\title{
Integrated water quality monitoring of Mahi river using benthic macroinvertebrates and comparison of its biodiversity among various stretches
}

\author{
Nitasha Khatri ${ }^{1} \mathbb{D} \cdot$ Krutarth Raval $^{1} \cdot$ Ashutosh K. Jha ${ }^{1}$
}

Received: 24 February 2020 / Accepted: 11 June 2021 / Published online: 26 July 2021

(c) The Author(s) 2021

\begin{abstract}
Use of benthic macroinvertebrates has been in vogue as indicator organisms for water quality monitoring since long. Traditional methods of water quality monitoring incorporate mostly monitoring of physicochemical parameters. However, it is the biomonitoring studies that could help in more accurate water quality monitoring. Due to anthropogenic impact at water sources, integrated water quality monitoring has become a necessity. An integrated study comprising physicochemical parameters and biomonitoring using benthic macroinvertebrates was carried out at six sites along the Mahi river basin, the command area of which has witnessed tremendous economic and cultural progress over a period of time. Diversity Score and Saprobic Score were calculated, and Water Quality Class was determined as per Biological Monitoring Working Party score given by Central Pollution Control Board and results in moderate pollution at all locations, whereas Water Quality Class as per ISI-IS 2296-1982 at M-1, M-4, M-7 and M-12 shows slight pollution. Jaccard's similarity index compared the biodiversity of benthic macroinvertebrates at all the sites from upstream to downstream locations. It was maximum, i.e., 52.94\% between the locations at M-4 and M-7. Species richness (S) has also been monitored at the said locations. The Diversity Score 0.83 is maximum at the Galteshwar (M-12) location of the Mahi river, which indicates that the location was the most ecologically diverse region among all.
\end{abstract}

Keywords Diversity Score $\cdot$ Saprobic Score $\cdot$ Jaccard similarity index $\cdot$ Water quality $\cdot$ Aquatic $\cdot$ Species richness

\section{Introduction}

One of the basic needs of all living beings is access to clean fresh water. A river normally serves as the vital source for survival as several activities, besides use such as drinking water, farming, electricity generation, industrialization. These activities, however, lead to contamination of water bodies besides causing organic and inorganic river water pollution (Khatri et al. 2016a, 2017a; Khatri et al. 2020a, b, c, d; Khatri and Tyagi 2015a; Rawtani et al. 2018; Tyagi et al. 2018).

There exist two types of pollution in water: chemical and biological. Benthic macroinvertebrates act as indicator of

Nitasha Khatri

nitashakhatri1983@gmail.com

Department of Forest and Environment, Gujarat Environment Management Institute, Jivraj Mehta Bhavan, Sector 10B, Gandhinagar, Gujarat, India pollution that help to detect the impact of anthropogenic activities on the ecosystem: aquatic and terrestrial (Khatri et al. 2017b; Khatri et al. 2020a, b, c, d; Parikh et al. 2020; Patel et al. 2021).

Benthic macroinvertebrates are majorly insects that dwell on the floor of water bodies. They either spend their entire lives in water or grow up to become terrestrial insects. They are found in all water bodies, as they have a wide range of pollution tolerance among various species. The benthic macroinvertebrates community structure depends on the exposure to pollution it receives (Bhadrecha et al. 2016; Khatri et al. 2020a, b, c, d; Pimparkar et al. 2016).

Benthic macroinvertebrates have been used as indicator organisms to measure the water quality of water bodies across the world (Aazami et al. 2015; Bowles et al. 2017; Giri and Singh 2012; Lock et al. 2011; M. P. Sharma et al. 2008). Studies have been carried out in India on certain rivers such bioassessment of river Ujh using benthic macroinvertebrates (Singh et al., 2019), in 2017, while Central Pollution Control Board had carried out one such study on 
benthic macroinvertebrate of the River Ganga (CPCB 2017). The understanding of the distribution pattern of benthic macroinvertebrate and niche organization is mandatory for ecological assessment of river. This change in the ecological pattern of macrobenthos distribution by different anthropogenic activities is reported from the Teesta river and Ganga river from the Himalayas-India (Bhatt \& Pandit 2010; Nautiyal 2010), Vindhyan river and Plateau river from Central highlands-India (Mishra \& Nautiyal 2011, 2013) and different stretches of Western Ghats-India (Subramanian \& Sivaramakrishnan 2005).

Ninglad stream and Behta river located in India were assessed by the researchers using the benthic macroinvertebrate diversity and through physiochemical parameters to cross-verify the pollution levels. They employed the Nepalese Biotic Score (NEPBIOS) and National Sanitation Foundation Water Quality Index (NSFWQI) to analyze the water quality. Both the mentioned index used the benthic macroinvertebrate community data combined with water quality parameters (Sharma et al. 2006, 2008). Mishra and Pandey (2019) have worked on the structural and functional condition of Macrobenthos fauna of the major rivers Dhasan, Ken and Tons located at the central highlands ecoregionIndia. They revealed that the establishment of the benthic macroinvertebrate community was influenced by manmade activities like scraper, shredder and collector effects (Mishra \& Pandey 2019). Asan, Suswa and Tawa streams of Doon valley were also explored to identify the assemblage of benthic macroinvertebrate that has been driven by the ecological parameters (Mishra et al. 2020).

A study was carried out on Mahi river mapped variation on Water Quality Index during Monsoon season using Geographical Information System, which revealed that the study area, i.e., southeast of Anand and northwest of Vadodara, is a highly polluted zone (Srivastava et al. 2011). Even assessment of seasonal variation using phytoplankton has been carried out on the Mahi River, which indicated average ecological condition pointing toward correlation between physicochemical parameters and density of phytoplankton and zooplankton (N. K. Sharma and Bhardwaj 2011), whereas an integrated study of benthic macroinvertebrates and physicochemical parameters carried out in 2015 of Mahi River near Galteshwar revealed that the water was slightly polluted (Bhadrecha et al. 2016; Khatri et al. 2020a, b, c, d). This study comprises of an integrated study of biomonitoring and physicochemical monitoring of the River Mahi for overall and accurate assessment of water quality of river stretch in Gujarat.

The selection of this river for the study was based on the fact that while the banks of the river have witnessed tremendous growth in economic and cultural activities, the stretches along the river have seen enhanced pollution that needs to be monitored and assessed periodically.
Rivers carry alluvial soils, which provide the most productive agricultural lands across the country e.g., the fertile Indo-Gangetic plain, and the Ganga-Brahmaputra, and the Cauvery delta (Dobrovol'ski, 2011). Besides being the source of water, rivers also dilute the contaminants and flush them out, thus acting as a profuse cleaner for towns and cities. Furthermore, rivers do act as primary channels of inland waterways and also cause formation of flatlands due to deposition of sediments. The rivers are the major source of sand used in construction including in dams. The construction of dams leads to power generation, besides its use for recreation, tourism and fishing activities. Several dams have come up in the Mahi basin, Viz. Panam Dam, Mahi Bajaj Sagar Project and Kadana Dam. Jakham Reservoir (Gor and Shah 2014). Among these large dams/ reservoirs, Mahi Bajaj Sagar dam and Kadana Dam generate hydropower, while several other medium- to smallscale hydroprojects primarily cater to the irrigation and industrial needs (Tortajada 2014).

Water quality assessment of the Mahi river is carried out using four different biostatical indices as mentioned hereinafter. Biomonitoring water quality criteria are analyzed with the help of BMWP score values and Diversity Score values. Both values give a result that indicates the quality of the riverine system. Jaccard similarity index is used to identify the similarity between the selected sample sites and the diversity of that area. The species richness index is solely focused on the number of families present at the location. The result value would indicate the ecological quality of that area that may be useful for environmental impact assessment as also to have comparison of river quality after COVID spread so as to ascertain whether this has been any change in the ecological aspect of the river.

The richness is the number of species per sample,

\section{Study area}

In Gujarat, Mahi river flows from Kadana reservoir ( $23^{\circ} 31^{\text {' }}$ $\left.\mathrm{N} 73^{\circ} 83^{\prime} \mathrm{E}\right)$ to Kamboi $\left(22^{\circ} 21^{\prime} \mathrm{N} 72^{\circ} 61^{\prime} \mathrm{E}\right)$ with a drainage area of $11,694 \mathrm{~km}^{2}$. The entire stretch was surveyed for potential sampling location based on land use, accessibility and anthropogenic activities. Nineteen sampling locations were selected and numbered from M-1 to M-19, Table 1 . Pre-monsoon sampling was carried out at these locations from April to May 2019. Sampling was possible only at locations mentioned in Fig. 1, while the river was dried up at locations M-5, M-10, M-11, M-13 and M-14: Further, farming was being done in the vicinity of locations M-2 and M-16 making the river bank not accessible, whereas sampling at M-6, M-9, M-16, M-17 was not possible due to deep waters at such places. The river was brackish and almost dried up at M-18, whereas M-19 was crammed with crabs. 
Table 1 Locations of sampling

\begin{tabular}{llll}
\hline Location code & Location name & Surrounding activities & Uses of water \\
\hline M-1 & Kadana Dam & Fishing, Religious Discard, Transportation & $\begin{array}{c}\text { Domestic, Drinking, Irrigation } \\
\text { and Hydropower generation } \\
\text { Domestic, Irrigation } \\
\text { Immersion of Idols (occasionally) }\end{array}$ \\
M-4 & Limbodara, Mahisagar Bridge & Bathing, Washing, Religious Discard, & Domestic, Irrigation \\
M-7 & Hadod Bridge, Mahisagar & Construction of Bridge & $\begin{array}{l}\text { Domestic, Irrigation } \\
\text { Immersion of Idols (occasionally) }\end{array}$ \\
M-8 & Sevaliya-Godhra Road-1 & Bathing, Washing, Mining & $\begin{array}{l}\text { Domestic, } \\
\text { Immersion of Idols (occasionally) }\end{array}$ \\
M-12 & Sevaliya-Godhra Road-2 & Bathing, Washing, & Domestic, \\
M-15 & Galteshwar & Bathing, Washing, Religious Discard, Crematoria activities, & Immersion of Idols (occasionally) \\
& Savli - Ode Bridge & Bathing, Washing, Construction activities & Domestic, Irrigation \\
M-16 & Mahisagar Bridge & Surrounded by Forest, No anthropogenic activities were & Domestic, Irrigation \\
M-17 & Umeta Bridge & observed & Domestic, \\
M-18 & Bamangam & Washing, Bathing & Immersion of Idols (occasionally) \\
M-19 & Kamboi & No activity observed & No use ( estuary) \\
\hline
\end{tabular}

Fig. 1 Sampling locations of Mahi river

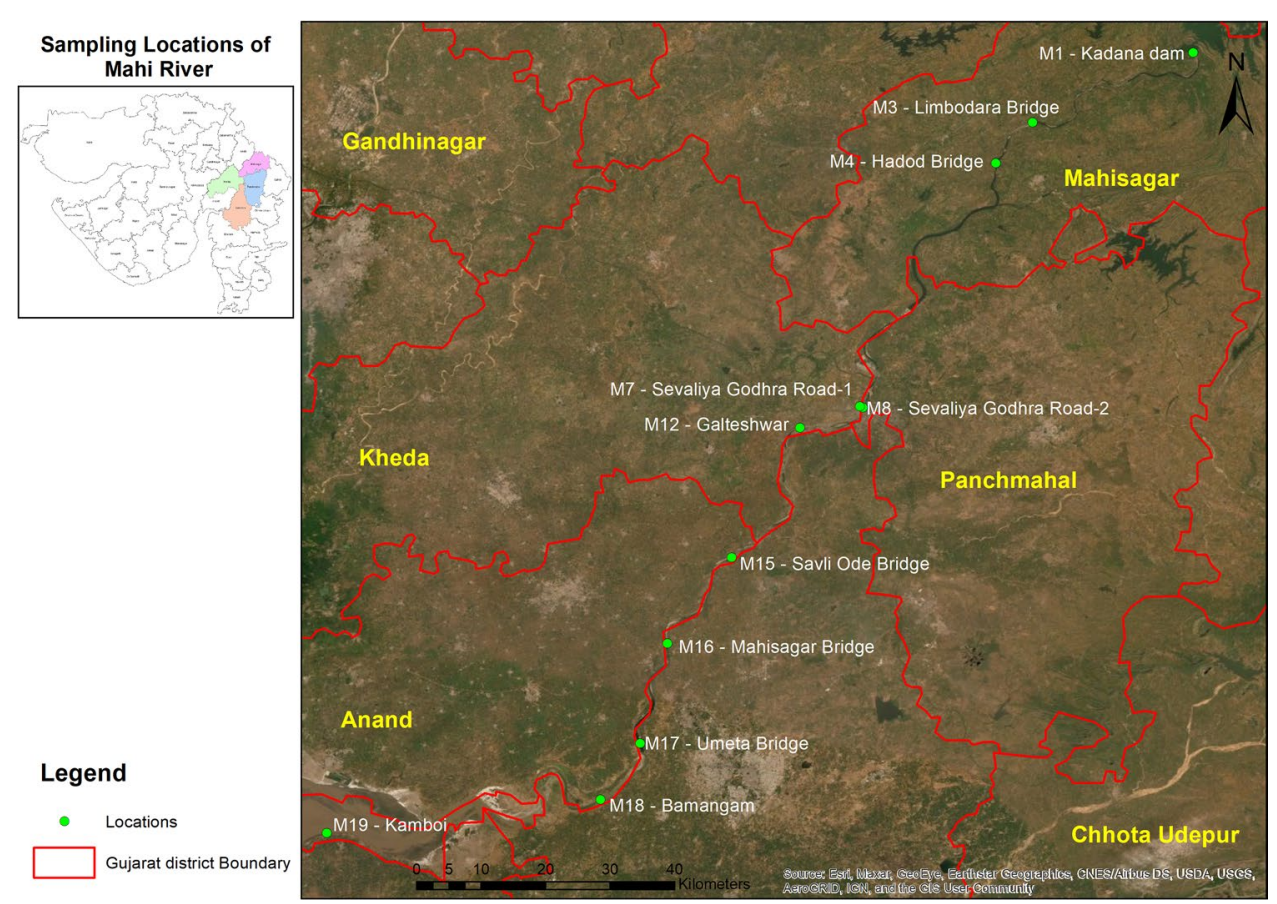

\section{Materials and methods}

\section{Sampling of benthic macroinvertebrates}

Different habitats support different densities of specific organisms. Each location was covered for a length of $500 \mathrm{~m}$. A random sampling of benthic macroinvertebrates was performed according to the standard procedure developed in Biological Laboratory, Central Pollution Control Board, using hand net, D net, shovel sampler, hand gloves, forceps, scientific sieves and wide mouth bottles. Depending upon the nature of the substratum of the water body, different sampling devices and methods were used. The sampled organisms were preserved in a 250-mL-wide mouth polyethylene bottle containing $4 \%$ formalin solution and brought to the laboratory for identification of organisms up to family taxon and further used 
for the determination of Biological Monitoring Working Party (BMWP) or Saprobic Score and Diversity Score.

Saprobic Score or BMWP gives a numerical value that includes species diversity and ecological sensitivity of individual taxa. The number of pollution-sensitive macroinvertebrates and the number of taxonomic groups tend to decrease with increase in pollution. All possible families are allocated Saprobic value classified on the score scale of 1 to 10 according to their preference for Saprobic water quality. The families which are most sensitive to pollution such as Leptophlebiidae (Mayflies), are allocated a score of 10, whereas tolerant families like Oligochaete (worms) 1 . The sum of the multiplied score of each family is divided by the total number of families in sample to calculate Saprobic Score.

Saprobic Score $=\frac{\text { Grand total of multiplied score }}{\text { Grand total of number of families encountered }}$

The Diversity Score is based on the numerical abundance of each species. Stable ecosystems generally have high species diversity. The changes in environmental conditions affect the community structure. Anthropogenic stress or natural disturbances affect the diversity of a region. Diversity Score is calculated by dispersing sampled specimens of benthic macroinvertebrates in a plastic tray with a 1 inch $\times 1$ inch grid as shown in Fig. 2. A sequential pair-wise comparison is made from left to right, starting from the top left grid and ending at the bottom right grid. The organism in current square is marked 1 if it is different from an organism in the previous square and is marked 0 if the same. Each comparison step is called run.

Diversity score $=\frac{\text { Total number of runs }}{\text { Total number of organisms }}$
Jaccard's index (J) compares biodiversity levels across sites in terms of richness component and not abundance. It was studied how Jaccard's index varied with the distance or environmental differences between two sites.

$J=100 \frac{T c}{(T 1+T 2)}$

where $\mathrm{Tc}$ is the number of common families encountered between site 1(T1) and site 2(T2) (Jaccard 1908).

This index generates a value between 0 and 1 . The result value will give the estimation of the similarity between sites.

Species richness $(\mathrm{S})$ is the total number of different organisms present in an ecological community or region (Robert 2009).

\section{Sampling of river water for physicochemical analysis}

The methods described in GEMI's Sampling Protocol and 'Standard Methods for Examination of Water and Waste Water,' 23rd Edition as prescribed by American Public Health Association (APHA) was adopted for the sampling of river water and analysis of parameters considered, namely pH, Turbidity, Total Dissolved Solids, Ammoniacal Nitrogen, COD, BOD, DO, Total Alkalinity, Total Hardness, Calcium Hardness, Magnesium Hardness, Chloride, Fluoride, Sulfate, Nitrate, Phenol, Sodium Adsorption Ratio and Oil and Grease, along with detection of heavy metals such as Fe, $\mathrm{Zn}, \mathrm{As}, \mathrm{Cu}, \mathrm{Pb}, \mathrm{Cd}, \mathrm{Ni}, \mathrm{Cr}$ and $\mathrm{Mn}$. The bacterial parameters analyzed were Total Coliform and Fecal Coliform. Necessary preservation procedures were followed for the required parameters according to APHA $23^{\text {rd }}$ Edition. Preserved samples were further analyzed at GEMI's laboratory. Samples were collected and analyzed in duplicate, and averaged values were considered.
Fig. 2 Biological assessment of benthic macroinvertebrates in laboratory

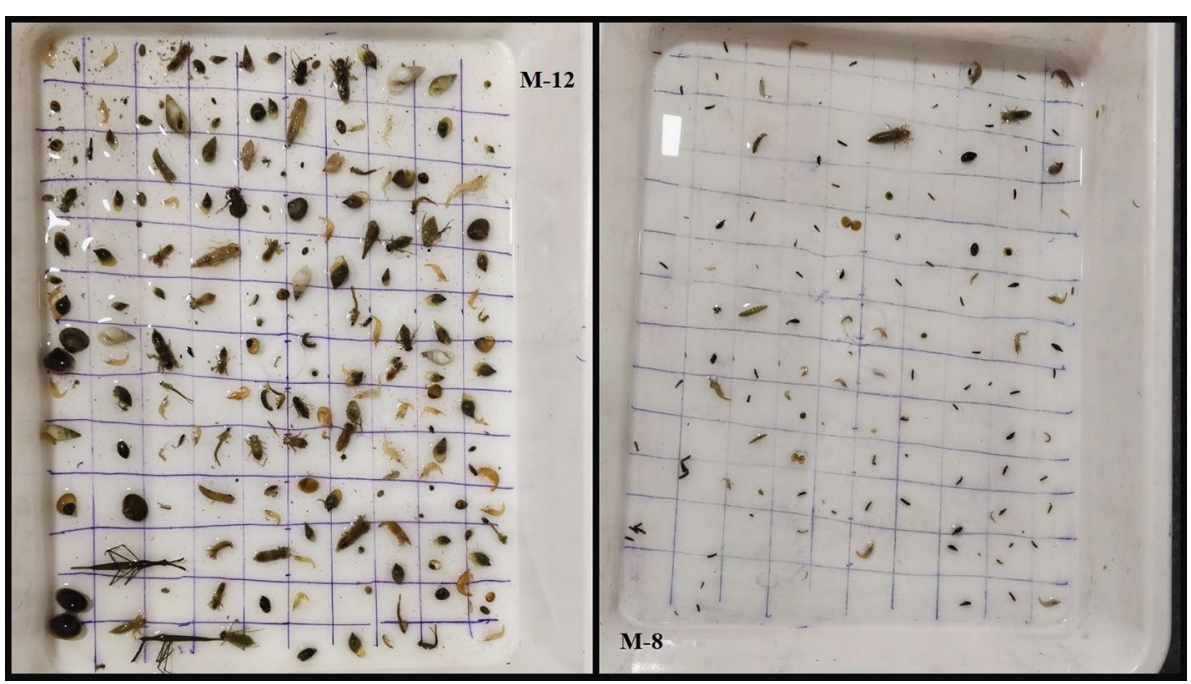




\section{Results and discussion}

\section{Assessment of benthic macroinvertebrates}

Benthic macroinvertebrates sampled at locations M-1, M-3, M-4, M-7, M-8, M-12 and M-15 were identified up to family level, Fig. 3, and their Saprobic Score and Diversity Score were calculated.

The Saprobic Score and Diversity Score were compared to Biological Water Quality Criteria (BWQC) derived by CPCB, Table 2, to identify the Water Quality Class of each location, which is summarized in Table 3. However, the detailed identification and calculation for all locations depicted in Table 4 and Table 5 show the families of macroinvertebrates of pollution-sensitive and/ or tolerant species in the studied locations together.

The Saprobic and Diversity Score values indicate the Water Quality of all sampled locations is 'Class C-Moderate Pollution.' Class $\mathrm{C}$ represents the narrow range of diversity for organisms with low Saprobic value allowing an abundance of pollution-tolerant species.

Each site was studied with respect to the number of families at each BMWP score (1 to 10). No significant changes were observed in the number of pollution-sensitive families with Saprobic Value of 10 . The number of families with a Saprobic Score of 8 gradually increased as the river flowed from upstream to downstream. It was observed that the
Fig. 3 Identification of organisms sampled at location a M-12 and b M-8, up to family taxa
Table 2 Biological Water Quality Criteria (BWQC) developed by CPCB

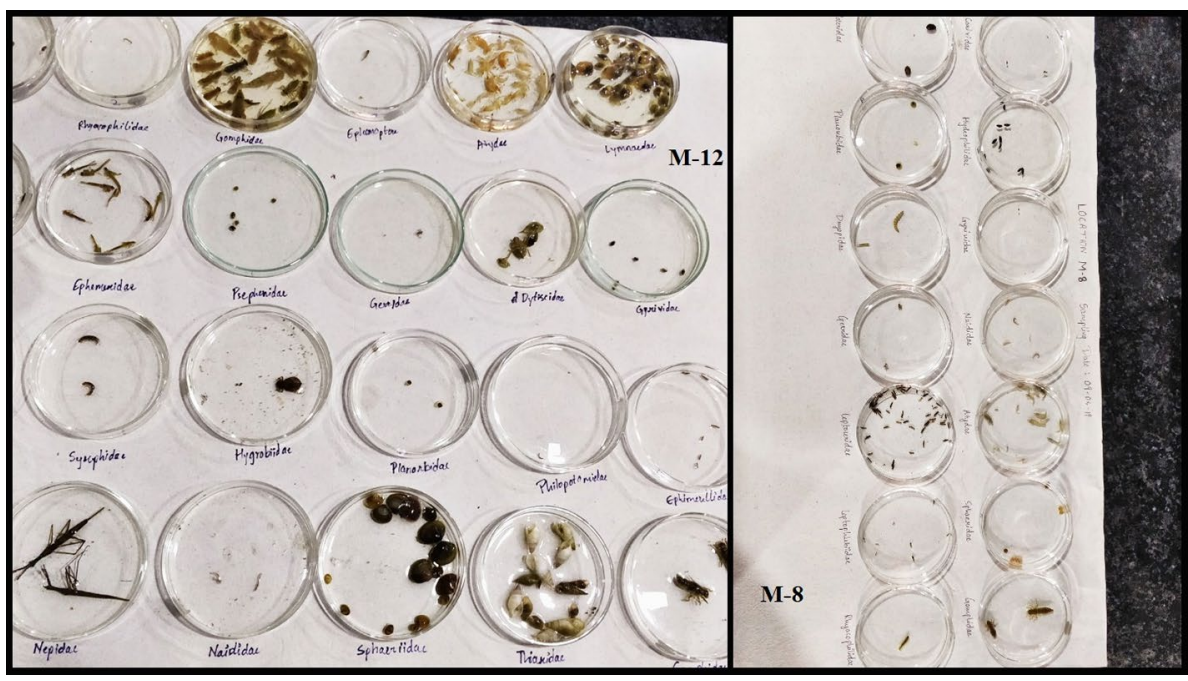

\begin{tabular}{lllll}
\hline $\begin{array}{l}\text { Range of Saprobic } \\
\text { Score }\end{array}$ & $\begin{array}{l}\text { Range of Diversity } \\
\text { Score }\end{array}$ & Water quality & $\begin{array}{l}\text { Water Quality } \\
\text { Class }\end{array}$ & Indicator color \\
\hline 7 and more & $0.2-1.0$ & Clean & A & Blue \\
$6-7$ & $0.5-1.0$ & Slight Pollution & B & Light Blue \\
$3-6$ & $0.3-0.9$ & Moderate Pollution & C & Green \\
$2-5$ & $0.4-$ Less & Heavy Pollution & D & Orange \\
$0-2$ & $0-0.2$ & Severe Pollution & E & Red \\
\hline
\end{tabular}

Table 3 Water Quality Class of Mahisagar River based on BWQC

\begin{tabular}{llllllll}
\hline $\begin{array}{l}\text { Location } \\
\text { Parameter }\end{array}$ & M-1 & M-3 & M-4 & M-7 & M-8 & M-12 & M-15 \\
\hline Saprobic Score & 5.375 & 5.222 & 5.33 & 5.571 & 5.944 & 5.542 & 5.566 \\
Diversity Score & 0.76632 & 0.5115 & 0.7040 & 0.7065 & 0.6904 & 0.83925 & 0.6959 \\
Water Quality Class & C & C & C & C & C & C & C \\
Water Quality & Moderate Pollution & Moderate & Moderate & Moderate & Moderate & Moderate Pollution & Moderate \\
& & Pollution & Pollution & Pollution & Pollution & & Pollu- \\
& & & & & & &
\end{tabular}


Table 4 Locationwise calculation of Saprobic Score

\begin{tabular}{|c|c|c|c|c|c|}
\hline Taxonomical group & Taxonomical families & $\begin{array}{l}\text { Mark encountered families and if } \\
\text { possible species within families also } \\
\text { mark abundancy AS - A, B, C, D, E }\end{array}$ & Total families & BMWP Score & $\begin{array}{l}\text { Multi- } \\
\text { plied } \\
\text { score }\end{array}$ \\
\hline \multicolumn{6}{|c|}{ LOCATION M-1: KADANA DAM } \\
\hline Odonata & Gomphidae & $\mathrm{B}$ & 1 & & \\
\hline \multicolumn{4}{|c|}{ Total families encountered \& total multiplied score } & $1 \times 8$ & 8 \\
\hline \multirow[t]{3}{*}{ Mollusca } & Thiaridae & $\mathrm{B}$ & 3 & & \\
\hline & Bithynidae & $\mathrm{C}$ & & & \\
\hline & Unionidae & $\mathrm{B}$ & & & \\
\hline Crustacea & Atydae & $\mathrm{C}$ & 1 & & \\
\hline \multicolumn{3}{|c|}{ Total families encountered $\&$ total multiplied score } & & $4 \times 6$ & 24 \\
\hline Hemiptera & Corixidae & B & 1 & & \\
\hline Coleoptera & Gyrinidae & $\mathrm{C}$ & 1 & & \\
\hline \multicolumn{4}{|c|}{ Total families encountered \& total multiplied score } & $2 \times 5$ & 10 \\
\hline Oligochaeta & All families & A & & $1 \times 1$ & 1 \\
\hline \multicolumn{4}{|c|}{$\begin{array}{l}\text { Total families encountered \& total } \\
\text { multiplied score }\end{array}$} & $1 \times 1$ & 1 \\
\hline \multicolumn{3}{|c|}{ Grand total families encountered $\&$ grand total multiplied score } & 8 & & 43 \\
\hline \multicolumn{6}{|c|}{ LOCATION M-3: LIMBODRA, MAHISAGAR BRIDGE } \\
\hline Trichoptera & Leptoceridae & $\mathrm{D}$ & 1 & & \\
\hline \multicolumn{4}{|c|}{ Total families encountered \& total multiplied score } & $1 \times 10$ & 10 \\
\hline \multirow[t]{2}{*}{ Mollusca } & Thiaridae & A & 2 & & \\
\hline & Bithynidae & $\mathrm{B}$ & & & \\
\hline Crustacea & Atydae & A & 1 & & \\
\hline \multicolumn{3}{|c|}{ Total families encountered \& total multiplied score } & & $3 \times 6$ & 18 \\
\hline \multirow[t]{2}{*}{ Coleoptera } & Gyrinidae & $\mathrm{B}$ & 2 & & \\
\hline & Hydrophilidae & A & & & \\
\hline \multicolumn{4}{|c|}{ Total families encountered \& total multiplied score } & $2 \times 5$ & 10 \\
\hline \multirow[t]{3}{*}{ Mollusca } & Lymnaeidae & $\mathrm{B}$ & 3 & & \\
\hline & Planorbidae & B & & & \\
\hline & Sphaeridae & B & & & \\
\hline \multicolumn{4}{|c|}{ Total families encountered \& total multiplied score } & $3 \times 3$ & 9 \\
\hline \multicolumn{3}{|c|}{ Grand total families encountered \& grand total multiplied score } & 9 & & 47 \\
\hline \multicolumn{6}{|c|}{ LOCATION M-4: HADOD BRIDGE, MAHISAGAR } \\
\hline Ephemeroptera & Leptophlebiidae & $\mathrm{C}$ & 1 & & \\
\hline \multirow{2}{*}{\multicolumn{3}{|c|}{$\begin{array}{l}\text { Trichoptera Leptoceridae } \\
\text { Total families encountered \& total multiplied score }\end{array}$}} & 1 & & \\
\hline & Total families encountered \& total multiplied score & & & $2 \times 10$ & 20 \\
\hline Odonata & Gomphidae & B & 1 & & \\
\hline \multicolumn{3}{|c|}{ Total families encountered \& total multiplied score } & & $1 \times 8$ & 8 \\
\hline \multirow[t]{2}{*}{ Mollusca } & Viviparidae & $\mathrm{B}$ & 2 & & \\
\hline & Bithynidae & $\mathrm{B}$ & & & \\
\hline Crustacea & Atydae & $\mathrm{C}$ & 1 & & \\
\hline \multicolumn{3}{|c|}{ Total families encountered \& total multiplied score } & & $3 \times 6$ & 18 \\
\hline Coleoptera & Gyrinidae & $\mathrm{B}$ & 2 & & \\
\hline & Noteridae & A & & & \\
\hline Total families encou & tiplied score & & & $2 \times 5$ & 10 \\
\hline Mollusca & Planorbidae & $\mathrm{B}$ & 2 & & \\
\hline & Sphaeridae & $\mathrm{B}$ & & & \\
\hline Total families encou & tiplied score & & & $2 \times 3$ & 6 \\
\hline Oligochaeta & All families & A & 2 & & \\
\hline Total families encou & tiplied score & & & $2 \times 1$ & 2 \\
\hline
\end{tabular}


Table 4 (continued)

Taxonomical group

Taxonomical families

Mark encountered families and if possible species within families also mark abundancy AS - A, B, C, D, E

Total families BMWP Score Multiplied score

Grand total families encountered \& grand total multiplied score 12

\section{LOCATION M-7: SEVALIYA - GODHRA ROAD-1}

Ephemeroptera

Leptophlebiidae

Trichoptera

Leptoceridae

Total families encountered \& total multiplied score

Odonata

Gomphidae

Total families encountered \& total multiplied score

Trichoptera

Rhyacophilidae

Total families encountered \& total multiplied score

Crustacea

Atydae

Total families encountered \& total multiplied score

Hemiptera

Gerridae

Corixidae

Coleoptera

Gyrinidae

Hydrophilidae

Dryopidae

Noteridae

Total families encountered \& total multiplied score

Mollusca

Planorbidae

Sphaeridae

Total families encountered \& total multiplied score

\section{Oligochaeta}

All families

Total families encountered \& total multiplied score

Grand total families encountered \& grand total multiplied score

\section{LOCATION M-8: SEVALIYA - GODHRA ROAD-2}

Ephemeroptera Leptophlebiidae

Trichoptera

Leptoceridae

Total families encountered \& total multiplied score

Odonata

Platycnemididae

Gomphidae

Total families encountered \& total multiplied score

Trichoptera Rhyacophilidae

Total families encountered \& total multiplied score

Mollusca

Viviparidae

Thiaridae

Bithynidae

Unionidae

Crustacea

Atydae

Total families encountered \& total multiplied score

\section{Hemiptera}

Gerridae

Coleoptera

Dytiscidae

Gyrinidae

Noteridae

Trichoptera

Hydropsychidae

Total families encountered \& total multiplied score

Mollusca

Lymnaeidae

Planorbidae

Sphaeridae
B

D

B

A

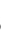

A

C

C

B

B

A

C

B

B

$6 \times 5 \quad 30$

B

B

2

$2 \times 3 \quad 6$

B

1

$\begin{array}{lll}1 \times 1 & 1 \\ & & \mathbf{7 8}\end{array}$

$\mathrm{C}$

1

1

$2 \times 10$

20

2

28

16

A

1

$1 \times 7 \quad 7$

4

$5 \times 6$

30

1

3

B

C

B

A

$5 \times 5$

25 
Table 4 (continued)

Taxonomical group
Taxonomical families

Mark encountered families and if possible species within families also mark abundancy AS - A, B, C, D, E

$\begin{array}{rr}\text { Total families } & \text { BMWP Score } \begin{array}{l}\text { Multi- } \\ \text { plied } \\ \text { score }\end{array}\end{array}$

$3 \times 3$

9

18

107

Grand total families encountered \& grand total multiplied score

8

LOCATION 12: GALTESHWAR

Ephemeroptera

Heptageniidae
Ephemerellidae
Ephemeridae

A

A

B

Total families encountered \& total multiplied score

\section{Odonata}

Gomphidae

$\mathrm{C}$

Trichoptera

Philopotamidae

A

Total families encountered \& total multiplied score

Trichoptera

Rhyacophilidae

A

Total families encountered \& total multiplied score

Mollusca

Viviparidae

Thiaridae

Crustacea

Atydae

Total families encountered \& total multiplied score

Hemiptera

Hydrometridae

Gerridae

Nepidae

Corixidae

Coleoptera

Hygrobidae

Dytiscidae

Gyrinidae

Hydrophilidae

Noteridae

Psephenidae

Total families encountered \& total multiplied score

Mollusca

Lymnaeidae

Planorbidae

Sphaeridae

Total families encountered \& total multiplied score

Diptera

Syrphidae

Total families encountered \& total multiplied score

Oligochaeta All families (Naididae) B

Total families encountered \& total multiplied score

Grand total families encountered \& grand total multiplied score

LOCATION M-15: SAVLI - ODE BRIDGE

Ephemeroptera Leptophlebiidae C

Total families encountered \& total multiplied score

Odonata

Plathycnemididae

Libellulidae

Trichoptera

Philopotamidae

Total families encountered \& total multiplied score

Trichoptera Rhyacophilidae

Total families encountered \& total multiplied score

Mollusca

Viviparidae

Thiaridae

Crustacea

Atydae
$10 \times 5$

50

3

$$
3 \times 3
$$

B
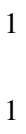

24

$$
1 \times 2
$$

1

C

$3 \times 8$

B

$1 \times 7$

7 
Table 4 (continued)

\begin{tabular}{|c|c|c|c|c|c|}
\hline Taxonomical group & Taxonomical families & $\begin{array}{l}\text { Mark encountered families and if } \\
\text { possible species within families also } \\
\text { mark abundancy AS - A, B, C, D, E }\end{array}$ & Total families & BMWP Score & $\begin{array}{l}\text { Multi- } \\
\text { plied } \\
\text { score }\end{array}$ \\
\hline \multicolumn{4}{|c|}{ Total families encountered \& total multiplied score } & $3 \times 6$ & 18 \\
\hline \multirow[t]{2}{*}{ Hemiptera } & Notonectidae & A & 2 & & \\
\hline & Corixidae & $\mathrm{C}$ & & & \\
\hline Coleoptera & Psephenidae & A & 1 & & \\
\hline Trichoptera & Hydropsychidae & B & 1 & & \\
\hline \multicolumn{4}{|c|}{ Total families encountered \& total multiplied score } & $4 \times 5$ & 20 \\
\hline \multirow[t]{3}{*}{ Mollusca } & Lymnaeidae & A & 3 & & \\
\hline & Planorbidae & B & & & \\
\hline & Sphaeridae & B & & & \\
\hline \multicolumn{4}{|c|}{ Total families encountered \& total multiplied score } & $3 \times 3$ & 9 \\
\hline Oligochaeta & All families & A & 1 & & \\
\hline \multicolumn{4}{|c|}{ Total families encountered \& total multiplied score } & $1 \times 1$ & 1 \\
\hline \multicolumn{3}{|c|}{ Grand total families encountered \& grand total multiplied score } & 16 & & 89 \\
\hline
\end{tabular}

Total families and multipiled score is used to calculate Saprobic score, these values for each location are mentioned in bold. By dividing multiplied score by families encountered at each location gives saprobic score

*Abundance Scale of Families: A=Single (One Individual), B=Scarce (2-10 Individuals), C=Common (10-50 Individuals), D=Abundant (50-100 Individuals), $\mathrm{E}=$ Excessive (more than 100 individuals)

\#The score is assigned as per BMWP score system designed by CPCB

number of moderate pollution-tolerant families with Saprobic values of 5 and 6 increased eloquently from location M-7 Godhra-Sevaliya Road -1 . This could be due to increased human interference and anthropogenic activities. On the contrary, the number of pollution-sensitive families (BMWP score-10,8) and pollution-tolerant families (BMWP score$2,1)$ showed no significant increase or decrease indicating toward the intermediate levels of pollution at the different sampling of locations, Fig. 4.

\section{Physicochemical analysis of river water}

The values of physicochemical parameters monitored were compared to IS:10,500:2012 Drinking Water Specification, Table 6 . The river water was slightly alkaline at all the locations. $\mathrm{pH}$ and turbidity of Mahi river exceeded the permissible limit at most of the locations and was as high as 9.59 and 914 NTU, respectively, at downstream locations, where river turns estuarine. Also, the values of Biochemical Oxygen Demand and Chemical Oxygen Demand were significantly high at downstream locations. Low Biochemical Oxygen Demand and high Dissolved Oxygen at location M-8 GodhraSevaliya-2 indicated good quality of water. Oil and grease were present postmiddle stream locations at M-8, M-12, M-16, M-17 and M-19 indicating the effect of anthropogenic activities. Levels of heavy metals such as Nickel, Zinc, Arsenic, Chromium, Lead and Copper were within acceptable limits, whereas Manganese exceeded the permissible limit at M-18 Bamangam and M-19 Kamboi. Levels of Cadmium and Iron exceeded the permissible limits throughout the river, but were significantly high at the downstream locations M-17, M-18 and M-19. Also, all the locations were found to be highly polluted by fecal coliforms.

Many anthropogenic activities carried out on the river banks justify the high level of water pollution (Khatri et al. 2016b; Khatri and Tyagi 2015b). Religious offerings, crematory activities and domestic activities are the major sources of organic pollution in the river. Furthermore, activities like mining and sand recovery use heavy machinery, which may contribute to oil and grease levels besides those contributed by motorboats and washing activities. The river banks contain nutrient-rich deposits, making them suitable for farming. Runoff from farming activities also contributes as nonpoint source of water pollution.

Water classification as per ISI-IS 2296-1982 has been adopted in India that specifies tolerance limits of parameters depending on various uses of water, Table 7 . The river water at all the locations was classified into Water Quality Class as per ISI-IS 2296-1982 based on values of physicochemical parameters. The locations M-1 Kadana Dam, M-4 Hadod Bridge, Mahisagar, M-7 Godra-Savli Road-1 and M-12 Galteshwar showed Water Quality of Class B, indicating that river water at these locations was fit for outdoor bathing. Locations M-3 Limbodra, Mahisagar, M-8 
Table 5 Availability of species in the taxonomical families at locations under study

\begin{tabular}{|c|c|c|c|c|c|c|c|c|c|}
\hline BMWP score & Taxonomical Families & M-1 & M-3 & M-4 & M-7 & M-8 & M-12 & M-15 & Pollution toleran \\
\hline \multirow[t]{5}{*}{10} & Ephemerellidae & & & & & & + & & \\
\hline & Ephemeridae & & & & & & + & & \\
\hline & Heptageniidae & & & & & & + & & \\
\hline & Leptoceridae & & + & + & + & + & & & \\
\hline & Leptophlebidae & & & + & + & + & & + & (1) \\
\hline \multirow[t]{5}{*}{8} & & & & & & & & & $\tilde{v}$ \\
\hline & Gomphidae & + & & + & + & + & + & & $\frac{c}{\sigma}$ \\
\hline & Libellulidae & & & & & & & + & 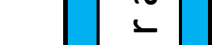 \\
\hline & Philopotamidae & & & & & & + & + & $\stackrel{0}{-1}$ \\
\hline & Plathycnemididae & & & & & + & & + & 0 \\
\hline 7 & Rhyacophilidae & & & & + & + & + & + & $\vdash$ \\
\hline \multirow[t]{5}{*}{6} & Atydae & + & + & + & + & + & + & + & \\
\hline & Bithyniidae & + & + & + & & + & & & $ᄃ$ \\
\hline & Thiaridae & + & + & & & + & + & & .0 \\
\hline & Unionidae & + & & & & + & & & \pm \\
\hline & Viviparidae & & & + & & + & + & + & 工 \\
\hline \multirow[t]{14}{*}{5} & Corixidae & + & & & + & & + & + & $\overline{0}$ \\
\hline & Dryopidae & & & & + & & & & 0 \\
\hline & Dytiscidae & & & & & + & + & & \\
\hline & Gerridae & & & & + & + & + & & مמ \\
\hline & Gyrinidae & + & + & + & + & + & + & & 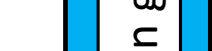 \\
\hline & Hydrometridae & & & & & & + & & $\bar{z}$ \\
\hline & Hydrophilidae & & + & & + & & + & & $n$ \\
\hline & Hydropsychidae & & & & & + & & + & (1) \\
\hline & Hygrobiidae & & & & & & + & & ড \\
\hline & Nepidae & & & & & & + & & $c$ \\
\hline & Noteridae & & & + & + & + & + & & 二 \\
\hline & Notonectidae & & & & & & & + & \\
\hline & Planariidae & & & + & & & & & \\
\hline & Psephenidae & & & & & & + & + & \\
\hline \multirow[t]{3}{*}{3} & Lymnaedae & & + & & & + & + & + & \\
\hline & Planorbidae & & + & + & + & + & + & + & \\
\hline & Sphaeridae & & + & + & + & + & + & + & \\
\hline 2 & Syrphidae & & & & & & + & & \\
\hline 1 & Naididae & + & & + & + & & + & + & \\
\hline
\end{tabular}

Godra-Savli Road-2, M-15 Savli-Ode Bridge and M-16 Mahisagar Bridge were highly contaminated by fecal coliform and showed Water Quality of Class C, i.e., drinking water source with conventional treatment but after disinfection. The downstream locations M-18 Bamangam and M-19 Kamboi showed high levels of Chloride, Magnesium Hardness, Total Dissolved Solids and Iron along with significantly high fecal coliforms which classified the river water quality in Class E, indicating that the water was suitable for irrigation, industrial cooling or controlled waste disposal.

The Water Quality Class as per Biological Water Quality Criteria (BWQC) and Water Quality Class as per ISIIS 2296-1982 were compared. The results of the physicochemical analysis are not in consonance with the results derived from Biological Water Quality Criteria developed by CPCB for every location, Table 8 . It was found that Water 
Fig. 4 Graphical representation of number of families encountered at sampling locations

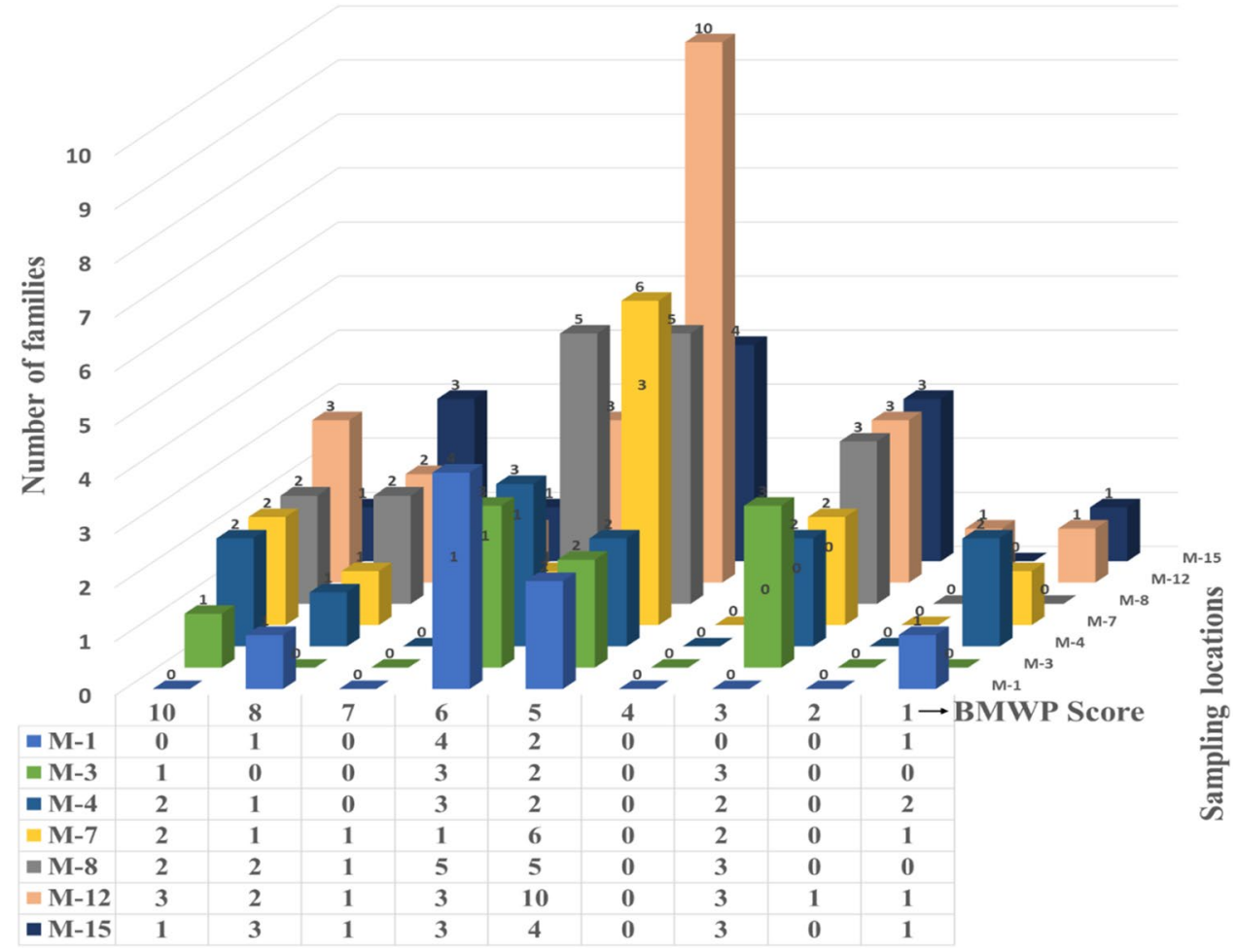

Table 6 Test result of physicochemical parameters of Mahi river and its comparison with IS:10,500:2012 Drinking Water Specification and Water Classification as per ISI-IS 2296-1982 depending on the use of water

\begin{tabular}{|c|c|c|c|c|c|c|c|c|c|c|c|c|c|c|}
\hline \multirow[b]{2}{*}{ PARAMETER } & \multirow[t]{2}{*}{ UNIT } & \multirow[t]{2}{*}{ M-1 } & \multirow[t]{2}{*}{ M-3 } & \multirow[t]{2}{*}{ M-4 } & \multirow[t]{2}{*}{ M-7 } & \multirow[t]{2}{*}{ M-8 } & \multirow[t]{2}{*}{ M-12 } & \multirow[t]{2}{*}{ M-15 } & \multirow[t]{2}{*}{ M-16 } & \multirow[t]{2}{*}{ M-17 } & \multirow[t]{2}{*}{ M-18 } & \multirow[t]{2}{*}{ M-19 } & \multicolumn{2}{|c|}{$\begin{array}{c}\text { IS:10500 } \\
\text { Drinking Water } \\
\text { Standard }\end{array}$} \\
\hline & & & & & & & & & & & & & $\mathbf{A L}$ & PL \\
\hline pH & - & 8.99 & 7.74 & 7.69 & 7.86 & 8.815 & 8.46 & 9 & 9.32 & 9.59 & 8.46 & 7.82 & $6.5-8.5$ & $6.5-8.5$ \\
\hline Turbidity & NTU & 157 & 6 & 6.3 & 4.2 & 2.2 & 5.067 & 0.2 & 0.3 & 9.32 & 746 & 914 & 1 & 5 \\
\hline Chloride & $\mathrm{mg} / \mathrm{L}$ & 23 & 26.17 & 28 & 70.83 & 32.29 & 86.45 & 32.88 & 37.44 & 95.97 & 15423.22 & 17267.14 & 250 & 1000 \\
\hline Total Hardness & $\mathrm{mg} / \mathrm{L}$ & 170 & 180 & 190 & 170 & 180 & 220 & 150 & 150 & 170 & 580 & 730 & 200 & 600 \\
\hline Calcium Hardness & $\mathrm{mg} / \mathrm{L}$ & 50 & 100 & 80 & 110 & 70 & 66.667 & 50 & 50 & 70 & 100 & 100 & - & - \\
\hline Magnesium Hardness & $\mathrm{mg} / \mathrm{L}$ & 120 & 80 & 110 & 60 & 110 & 153.33 & 100 & 100 & 100 & 480 & 630 & - & - \\
\hline $\begin{array}{c}\text { Total Alkalinity as } \\
\mathrm{CaCO}_{3}\end{array}$ & $\mathrm{mg} / \mathrm{L}$ & 163.54 & 185.78 & 224.99 & 202.1 & 174.43 & 328.05 & 136.5 & 147.28 & 121.34 & 116.4 & 125.53 & 200 & 600 \\
\hline Total Dissolved Solids & $\mathrm{mg} / \mathrm{L}$ & 188 & 225 & 258 & 320 & 227 & 450.67 & 238 & 258 & 356 & 28976 & 37106 & 500 & 2000 \\
\hline Ammoniacal Nitrogen & $\mathrm{mg} / \mathrm{L}$ & 0.58 & 0.58 & 0.58 & NIL & NIL & 0.46 & 0.58 & 0.58 & 0.47 & 0.45 & 0.47 & 0.5 & 0.5 \\
\hline COD & $\mathrm{mg} / \mathrm{L}$ & 36.5 & 27.6 & 19.7 & 14.81 & 8.39 & 19.2 & 35.4 & 36.4 & 19.89 & 1795.8 & 1676.94 & - & - \\
\hline Dissolved Oxygen & $\mathrm{mg} / \mathrm{L}$ & 8.36 & 3.66 & 4.14 & 2.65 & 7.915 & 6.376 & 6.25 & 6 & 6.66 & 7.25 & 7.75 & - & - \\
\hline BOD & $\mathrm{mg} / \mathrm{L}$ & 4.038 & 1.82 & 2.02 & 1.21 & 2.45 & 1.933 & 2.18 & 2 & 4.22 & 358.06 & 318.24 & - & - \\
\hline Oil \& Grease & $\mathrm{mg} / \mathrm{L}$ & NIL & NIL & NIL & NIL & 3.57 & 3 & NIL & 2.98 & 1 & NIL & 1.4 & - & - \\
\hline Fluoride & $\mathrm{mg} / \mathrm{L}$ & 0.32 & 0.533 & 0.546 & 0.777 & 0.679 & 0.862 & 0.762 & NIL & 0.359 & 1.011 & 1.116 & 1 & 1.5 \\
\hline Sulphate & $\mathrm{mg} / \mathrm{L}$ & 17.43 & 17.47 & 19.25 & 15.94 & 25.565 & 33.58 & 27.06 & 27.59 & 39.35 & 1948.15 & 2343.5 & 200 & 400 \\
\hline Nitrate & $\mathrm{mg} / \mathrm{L}$ & 2.65 & 2.51 & 2.78 & 2.37 & 2.605 & 10.416 & 2.79 & 4.04 & 2.87 & 9.39 & 4.79 & 45 & 45 \\
\hline SAR & milimole/L & 0.54 & 0.55 & 0.56 & 0.72 & 0.44 & 1.18 & 0.7 & 0.76 & 1.13 & 71.66 & 85.55 & - & - \\
\hline Phenol & $\mathrm{mg} / \mathrm{L}$ & $<0$ & $<0$ & $<0$ & 0.002 & 0.002 & 0.014 & $<0$ & $<0$ & $<0$ & 0.055 & 0.094 & 0.001 & 0.002 \\
\hline Nickel & $\mu \mathrm{g} / \mathrm{L}$ & 5.41 & 3.05 & 2.94 & 6.54 & 2.845 & 0.993 & 3.73 & 1.22 & 4.52 & 9.78 & 11.7 & 20 & 20 \\
\hline Zinc & $\mathrm{mg} / \mathrm{L}$ & 0.065 & 0.091 & 0.184 & 0.035 & 0.015 & 0.017 & 0.13 & 0.052 & 0.151 & 0.068 & 0.114 & 5 & 15 \\
\hline Cadmium & $\mathrm{mg} / \mathrm{L}$ & 0.35 & 0.01 & NIL & 0.02 & 0.14 & 0.23 & NIL & NIL & 0.74 & 0.25 & 0.21 & 0.003 & 0.003 \\
\hline Arsenic & $\mu \mathrm{g} / \mathrm{L}$ & 2.19 & 1.06 & 1.8 & NIL & 2.505 & 0.186 & 1.202 & 1.71 & 1.52 & 3.75 & 2.52 & 10 & 50 \\
\hline Chromium & $\mu \mathrm{g} / \mathrm{L}$ & 2.46 & 0.63 & 1.51 & 1.23 & 1.035 & 0.127 & 1.79 & 0.88 & 3.65 & 6.64 & 9.5 & 50 & 50 \\
\hline Lead & $\mu \mathrm{g} / \mathrm{L}$ & 3.48 & 2.18 & 3.32 & 1.82 & 0.895 & NIL & 8.34 & 8.93 & 8.85 & 6.18 & 6.05 & 10 & 10 \\
\hline Iron & $\mu \mathrm{g} / \mathrm{L}$ & 1297.52 & 270.36 & 458.5 & 91.54 & 119.8 & 71.453 & 85.06 & 103.2 & 543.09 & 4955.71 & 5985.6 & 300 & 300 \\
\hline Copper & $\mu \mathrm{g} / \mathrm{L}$ & 25.54 & 36.9 & 73.07 & 7 & 4.935 & 6.753 & 8.27 & 4.01 & 10.74 & 31.61 & 32.42 & 50 & 1500 \\
\hline Manganese & $\mu \mathrm{g} / \mathrm{L}$ & 84.21 & 160.24 & 176.95 & 36.56 & 32.365 & 50.006 & 74.37 & 30.02 & 66.15 & 389.35 & 507.85 & 100 & 300 \\
\hline Total Coliform & $\mathrm{MPN} / 100 \mathrm{ml}$ & 300 & 1600 & 1600 & 170 & 59 & 1600 & 300 & 1600 & 13 & 500 & 500 & Absent & Absent \\
\hline Fecal Coliform & MPN/100ml & 170 & 300 & 220 & 34 & 27 & 1600 & 80 & 350 & NIL & 70 & 80 & Absent & Absent \\
\hline $\begin{array}{c}\text { Water Classification as } \\
\text { per } \\
\text { ISI-IS 2296-1982 }\end{array}$ & 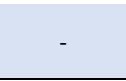 & B & C & B & B & $\mathbf{C}$ & B & $\mathbf{C}$ & C & $\mathbf{E}$ & $\mathbf{E}$ & $\mathbf{E}$ & - & - \\
\hline Above Acceptanc & Limit & & & & Abov & niss & imit & & & & & & & \\
\hline
\end{tabular}

$A L$ Acceptance Limit; PL Permissible Limit; $C O D$ Chemical Oxygen Demand; BOD Biochemical Oxygen Demand; SAR Sodium Adsorption Ratio 
Table 7 Use of water according to class as per ISI-IS 2296-1982

Table 8 Comparison of Water Quality Class as per BWQC and ISI-IS 2296-1982

\begin{tabular}{ll}
\hline Class A & Drinking water source without conventional treatment but after disinfection \\
\hline Class B & Outdoor bathing \\
Class C & Drinking water source with conventional treatment followed by disinfection \\
Class D & Fish culture and wildlife propagation \\
Class E & Irrigation, industrial cooling or controlled waste disposal \\
\hline
\end{tabular}

\begin{tabular}{llllllll}
\hline $\begin{array}{l}\text { Location } \\
\text { Parameter }\end{array}$ & M-1 & M-3 & M-4 & M-7 & M-8 & M-12 & M-15 \\
\hline $\begin{array}{l}\text { Water Quality } \\
\text { Class as per }\end{array}$ & $\begin{array}{c}\text { C Moder- } \\
\text { ate Pollu- } \\
\text { tion }\end{array}$ & $\begin{array}{c}\text { C Moder- } \\
\text { ate Pollu- } \\
\text { tion }\end{array}$ & $\begin{array}{c}\text { C Moder- } \\
\text { ate Pollu- } \\
\text { tion }\end{array}$ & $\begin{array}{c}\text { C Moder- } \\
\text { ate Pollu- } \\
\text { tion }\end{array}$ & $\begin{array}{l}\text { C Moder- } \\
\text { ate Pollu- } \\
\text { tion }\end{array}$ & $\begin{array}{c}\text { C Moder- } \\
\text { ate Pollu- } \\
\text { tion }\end{array}$ & $\begin{array}{c}\text { C Moder- } \\
\text { ate Pol- } \\
\text { lution }\end{array}$ \\
$\begin{array}{l}\text { Water Quality } \\
\text { Class as } \\
\text { per ISI-IS }\end{array}$ & B & C & B & B & C & B & C \\
2296-1982 & & & & & & & \\
\hline
\end{tabular}

Quality Class obtained at M-3 Limbodra, Mahisagar, M-8 Godra-Savli Road-2 and M-15 Savli-Ode Bridge was in consonance, i.e., showing water quality to be of moderate pollution that can be used as drinking water source with conventional treatment followed by disinfection. Locations M-1 Kadana Dam, M-4 Hadod Bridge, Mahisagar, M-7 Godra-Savli Road-1 and M-12 Galteshwar also showed moderate pollution with class $\mathrm{C}$ as per BWQC, and its chemical parameters showed Water Quality Class B, thereby making river water fit for outdoor bathing, but not for domestic uses (Khatri et al. 2017b).

\section{Calculation of Jaccard's similarity index}

By applying the formula as mentioned in Sect. 2, Jaccard's index was calculated from upstream to downstream location, between two simultaneous locations where biomonitoring was feasible.

It was revealed from Table 9 that the site that was closest to each other; i.e., locations M-7 and M-8 wherein the distance between them was less than a kilometer along the river, showed approx. 45\% Jaccard's similarity. However, the maximum similarity was observed between M-4 and M-7 locations despite they being maximum distance apart along the stretch which might be due to similarity in Water Quality Class as per IS 2296. This indicates that similar water quality of category B found at both M-3 and M-4 supports similarity index. Hence, it reveals that water quality correlates well with this index, and accordingly, as the water quality decreases from B to C from location M-7 to M-8, the similarity index also decreases. Species richness also
Table 9 Jaccard's similarity index values at different locations of the study area

\begin{tabular}{lll}
\hline Location & Distance & $\begin{array}{l}\text { Jaccard } \\
\text { similarity } \\
\text { index }\end{array}$ \\
\hline M-1 and M-3 & $33.26 \mathrm{~km}$ & $30.76 \%$ \\
M-3 and M-4 & $8.40 \mathrm{~km}$ & $31.25 \%$ \\
M-4 and M-7 & $50.22 \mathrm{~km}$ & $52.94 \%$ \\
M-7 and M-8 & $0.65 \mathrm{~km}$ & $45.45 \%$ \\
M-8 and M-12 & $9.98 \mathrm{~km}$ & $40.00 \%$ \\
M-12 and M-15 & $25.36 \mathrm{~km}$ & $29.03 \%$ \\
\hline
\end{tabular}

showed an increase from upstream to downstream up to location M-12, Fig. 5.

\section{Conclusion}

The various indices used to ascertain the water quality of the Mahi River reveals moderate pollution during the study period, which indicates that during the last three years, the river has become more polluted due to anthropogenic activities. However, in terms of biodiversity richness, river stream near the locations of Hadod Bridge Mahisagar (M-4) and Sevaliya-Godhra Road 1 (M-7) has maximum similarity in biodiversity of macroinvertebrates found there, which may result from similar water quality type. As a future prospect, there is need to monitor the diversity seasonally for trend analysis and statistical correlation. Such an effort will also ensure maintenance of water quality in dams on the Mahi 


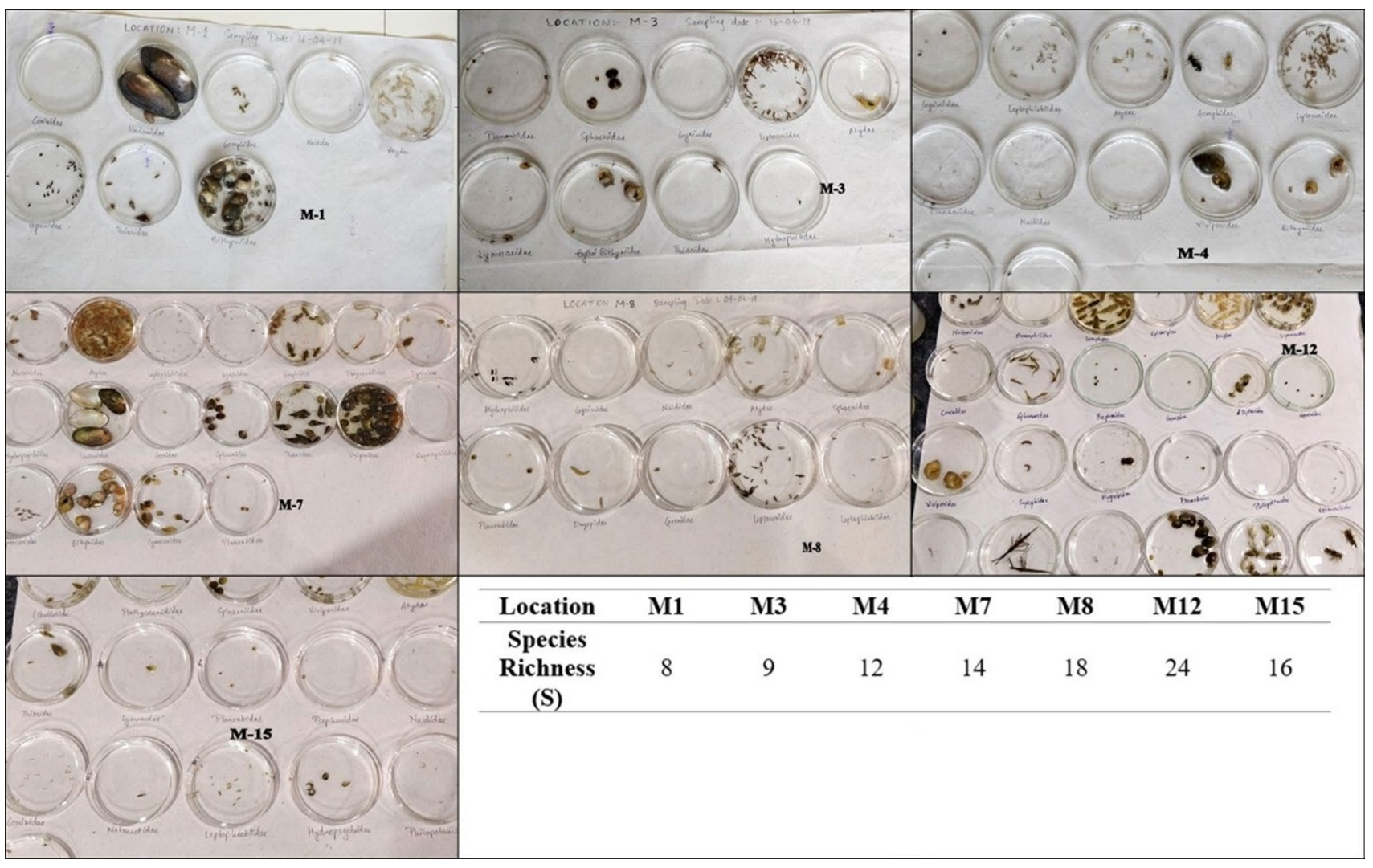

Fig. 5 Species richness at different locations of the study area

basin, which serves as a great source for irrigation and industrial needs. The Diversity Score being maximum at the Galteshwar (M-12) location of the Mahi river, makes it the most ecological diverse region among all locations.

Funding Author with file no- ECR/2017/001303 of Gujarat Environment Management Institute offers heartfelt thanks to Science \& Engineering Research Board, Ministry of Science \& Technology, Govt. of India, for Financial support.

\section{Declaration}

Conflict of Interest The authors declare that they have no conflict of interest.

Human and Animal Rights Research does not involve any human participants and animals. Hence ethical standards are taken care off.

Open Access This article is licensed under a Creative Commons Attribution 4.0 International License, which permits use, sharing, adaptation, distribution and reproduction in any medium or format, as long as you give appropriate credit to the original author(s) and the source, provide a link to the Creative Commons licence, and indicate if changes were made. The images or other third party material in this article are included in the article's Creative Commons licence, unless indicated otherwise in a credit line to the material. If material is not included in the article's Creative Commons licence and your intended use is not permitted by statutory regulation or exceeds the permitted use, you will need to obtain permission directly from the copyright holder. To view a copy of this licence, visit http://creativecommons.org/licenses/by/4.0/.

\section{References}

Aazami J, Esmaili-Sari A, Abdoli A, Sohrabi H, Van den Brink PJ (2015) Monitoring and assessment of water health quality in the Tajan River, Iran using physicochemical, fish and macroinvertebrates indices. J Environ Health Sci Eng 13(1):29. https://doi.org/ 10.1186/s40201-015-0186-y

Bhadrecha MH, Khatri N, Tyagi S (2016) Rapid integrated water quality evaluation of Mahisagar river using benthic macroinvertebrates. Environ Monit Assess 188(4):254. https://doi.org/10. 1007/s10661-016-5256-9

Bhatt JP, Pandit MK (2010) A macro-invertebrate based new biotic index to monitor river water quality. Curr Sci 99(22):196-203

Bowles DE, Hinsey JA, Cribbs T, Usrey FD, Morrison LW (2017) Long-term aquatic invertebrate monitoring at buffalo national river. Arkansas 71:16

CPCB (2017) Benthic Macroinvertebrates of River Ganga. Delhi: Central Pollution Control Board

Dobrovol'ski GV, Balabko PN, Stasjuk NV, Bykova EP (2011) Alluvial soils of river floodplains and deltas and their zonal differences Arid Ecosyst 1(3):119-124. https://doi.org/10.1134/S207909611 $103005 \mathrm{X}$ 
Giri N, Singh OP (2012) Bio-monitoring of water quality using benthic macro-invertebrates of river Singye Chhu in Bhutan. J Basic Appl Biol 6:44-49

Gor A, Shah MA (2014) Water quality index of mahi river Vadodara, Gujarat. J Engg Devel Res 2(3):6

Jaccard P (1908) Nouvelles recherches sur la distribution florale. Bull De La Société Vaudoise Des Sci Naturelles 44:223-270. https:// doi.org/10.5169/seals-268384

Khatri N, Tyagi S (2015) Influences of natural and anthropogenic factors on surface and groundwater quality in rural and urban areas. Front Life Sci 8(1):23-39. https://doi.org/10.1080/21553769. 2014.933716

Khatri N, Tyagi S, Rawtani D (2016a) Removal of basic dyes auramine yellow and auramine $\mathrm{O}$ by halloysite nanotubes. Int $\mathrm{J}$ Environ Waste Manag 17(1):44-59

Khatri N, Tyagi S, Rawtani D (2016b) Assessment of drinking water quality and its health effects in rural areas of Harij Taluka, Patan District of Northern Gujarat. Environm Claims J 28(3):223-246. https://doi.org/10.1080/10406026.2016.1190249

Khatri N, Tyagi S, Rawtani D (2017a) Recent strategies for the removal of iron from water: a review. J Water Process Eng 19:291-304

Khatri N, Tyagi S, Rawtani D (2017b) Rural environment study for water from different sources in cluster of villages in Mehsana district of Gujarat. Environ Monit Assess 190(1):10. https://doi. org/10.1007/s10661-017-6382-8

Khatri N, Raval K, Jha AK, Rawtani D (2020a) Pollution indicators at stretches of the Mahisagar River in Gujarat India. Environ Claims J 32(4):310-322

Khatri N, Tyagi S, Rawtani D, Tharmavaram M (2020b) Assessment of river water quality through application of indices: a case study River Sabarmati, Gujarat India. Sustain Water Resour Manag 6(6):101. https://doi.org/10.1007/s40899-020-00459-8

Khatri N, Tyagi S, Rawtani D, Tharmavaram M, Kamboj RD (2020c) Analysis and assessment of ground water quality in Satlasana Taluka, Mehsana district, Gujarat, India through application of water quality indices. Groundwater Sustain Develop 10:100321

Khatri N, Tyagi S, Tharmavaram M, Rawtani D (2020d) Sewage water: from waste to resource-a review. Environ Claims J. https://doi. org/10.1080/10406026.2020.1830585

Lock K, Asenova M, Goethals PLM (2011) Benthic macroinvertebrates as indicators of the water quality in Bulgaria: a case-study in the Iskar river basin. Limnologica 41(4):334-338. https://doi.org/10. 1016/j.limno.2011.03.002

Mishra AS, Nautiyal P (2011) Factors governing longitudinal variation in benthic macroinvertebrate fauna of a small Vindhyan river in Central Highlands ecoregion(central India). Trop Ecol 52(1):103-112

Mishra AS, Nautiyal P (2013) Functional composition of benthic macroinvertebrate fauna in the plateau rivers, Bundelkhand, central India. J Threat Taxa 5(13):4752-4758

Mishra AS, Pandey CK (2019) Structural and functional status of benthic macroinvertebrate fauna in the rivers of central highlands ecoregion. India Bioved 30(2):195-201
Mishra AS, Singh KR, Nautiyal P (2020) Factors governing distribution of benthic macroinvertebrates fauna in the springfed headwaters of Doon Valley India. J Mount Res 15:159-167

Nautiyal P (2010) Food chains of Ganga River ecosystems in the Himalayas. Aquat Ecosyst Health Manag 13(4):362-373

Parikh G, Rawtani D, Khatri N (2020) Insects as an indicator for environmental pollution. Environ Claims J. https://doi.org/10.1080/ 10406026.2020.1780698

Patel A, Rastogi N, Gandhi U, Khatri N (2021) Oxidative potential of atmospheric PM10 at five different sites of Ahmedabad, a big city in Western India. Environ Pollut 268:115909

Pimparkar M, Tyagi S, Khatri N, Rawtani D (2016) Development of criticality index to assess water quality in major rivers of Gujarat. Environ Claims J 28(4):320-345

Rawtani D, Khatri N, Tyagi S, Pandey G (2018) Nanotechnology-based recent approaches for sensing and remediation of pesticides. J Environ Manage 206:749-762. https://doi.org/10.1016/j.jenvm an.2017.11.037

Robert KC (2009) Biodiversity: concepts, patterns and measurement. In: Simon A, Levin (ed) The Princeton Guide to Ecology. Princeton University Press, Princeton, pp 257-263. http://assets.press. princeton.edu/chapters/s3_8879.pdf

Sharma NK, Bhardwaj S (2011) An assessment of seasonal variation in phytoplankton community of Mahi River (India). Geneconserve 10(40):154-164

Sharma MP, Sharma S, Gael V, Sharma P, Kumar A (2006) Water quality assessment of Behta River using benthic macroinvertebrates. Life Sci J 3(4):68-74

Sharma MP, Sharma S, Goel V, Sharma P, Kumar A (2008) Water quality assessment of Ninglad stream using benthic macroinvertebrates. Life Sci J 5(3):67-72

Singh V, Sharma MP, Sharma S, Mishra S (2019) Bio-assessment of River Ujh using benthic macro-invertebrates as bioindicators, India. Int J River Basin Manag 17(1):79-87

Srivastava PK, Mukherjee S, Gupta M, Singh SK (2011) Characterizing monsoonal variation on water quality index of River Mahi in India using geographical information system. Water Qual Expo Health 2(3-4):193-203. https://doi.org/10.1007/s12403-011-0038-7

Subramanian KA, Sivaramakrishnan KG (2005) Habitat and microhabitat distribution of stream insect communities of the Western Ghats. Current Sci 976-987

Tortajada C (2014) Dams: an essential component of development. J Hydrol Eng. https://doi.org/10.1061/(ASCE)HE.1943-5584. 0000919

Tyagi S, Rawtani D, Khatri N, Tharmavaram M (2018) Strategies for nitrate removal from aqueous environment using nanotechnology: a review. J Water Process Eng 21:84-95. https://doi.org/10.1016/j. jwpe.2017.12.005

Publisher's Note Springer Nature remains neutral with regard to jurisdictional claims in published maps and institutional affiliations. 